\title{
RADIATION FROM METALS BOMBARDED BY LOW.SPEED ELECTRONS
}

\author{
By F. L. Mohler and C. Boeckner
}

\section{ABSTRACT}

A small probe surface at a positive potential in a discharge may draw a current of many amperes per $\mathrm{cm}^{2}$ with the potential gradient limited to a thin sheath over the surface. Under such conditions a continuous spectrum is emitted by metal surfaces in caesium, potassium, and helium discharges. With a copper probe at 7 volts in caesium vapor, the intensity $J(\lambda)$ remains of the same magnitude between 6,200 and $2,400 \mathrm{~A}$ with a minimum at 5,800 and a maximum at 3,800 . Below 4 volts the spectrum has a high-frequency limit in this range (3,000 A for copper at 2.15 volts). On the assumption that this is analogous to a continuous X-ray spectrum, observed limits indicate work functions of 1.95 volts for $\mathrm{Cu}, 2.1$ for $\mathrm{Ag}, 1.45$ for $\mathrm{W}$ (all in caesium vapor). The absolute intensity (except for $\mathrm{Ag}$ ) agrees within a factor of 2 with values computed from the theoretical equation for intensity of the continuous X-ray spectrum. Efficiency for $\mathrm{Cu}$ at 7 volts is estimated as $5 \times 10^{-7}$. Comparison with published data on photo-electric effect of radiation from electron bombardment of metals indicates that the magnitude of effects below 10 volts can be,explained by this continuous spectrum.

\section{CONTENTS}

I. Introduction

II. Apparatus.... 674

III. Discharge conditions

IV. Location of radiation

V. Observations in various gases

VI. Vapor pressure

VII. Probe current

VIII. Spectral intensity distribution

IX. Intensity and voltage

X. Probe material

XI. Discussion

\section{INTRODUCTION}

There is some experimental evidence that the continuous X-ray spectrum extends on the long wave length side through the visible region. Lillienfeld and Rother ${ }^{1}$ have reported a bluish continuous spectrum emitted from the focal spot of an X-ray tube operated at X-ray voltages. Foote, Meggers, and Chenault ${ }^{2}$ found a continuous spectrum excited by bombarding metals with 1,000-volt electrons which extended throughout the range of a quartz spectrograph. The numerous studies ${ }^{3}$ of the photo-electric effect of the unresolved radiation from metals bombarded by lower-speed electrons include measurements with applied potentials as low as 3 volts. These effects probably come from both continuous radiation and characteristic line emission, and there is little evidence as to which is predominant.

1 Lillienfeld and Rother, Phys. Zeits., 21, p. 49; 1920; and correction note p. 360 ib.

2 Foote, Meggers, and Chenault, J. Opt. Soc. Am., 9, p. 541; 1924.

3 Holwesk, De la Lumière aux Rayons X; Univ. of France Press. 
Presumably a continuous spectrum in the visible could be excited by any potential above a few volts, but we would expect the efficiency at low voltage to be extremely low. It occurred to the authors that an effective means of obtaining high-current density at low voltage would be to use as a target a small probe surface in the highly ionized region of any discharge. Current densities as high as $10 \mathrm{amp}$ 's. $/ \mathrm{cm}^{2}$ can be obtained at any positive potential below the arcing potential, and with such currents the field produced by the applied potential is limited to a thin sheath over the surface of the order of $10^{-3} \mathrm{~cm}$ thick. Thus collisions in the sheath are negligible and electrons strike the surface with their full energy. An obvious complication is that the faint radiation from the metal must be viewed in the presence of a brilliant discharge.

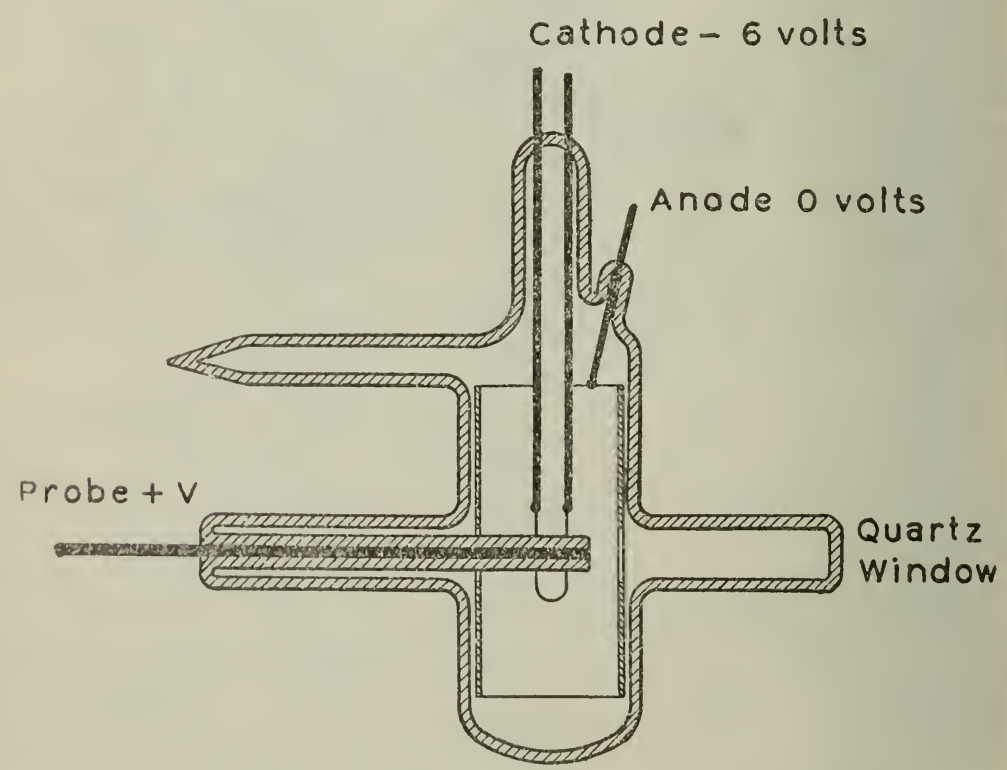

FICURE 1.-Type of tube used for observation of probe radiation

Trial spectrograms of a small wire probe at a positive potential of 7 or 8 volts in a caesium discharge, gave evidence of a continuous spectrum more or less masked in the visible by line emission, but conspicuous in the ultra-violet. This paper reports preliminary experiments on the various factors influencing the intensity of this radiation.

\section{APPARATUS}

A thermionic discharge was maintained between a cylindrical anode and a hairpin or helical cathode near the center of the cylinder. (Fig. 1.) The probe surface was the end of a heavy wire 1.5 to $3 \mathrm{~mm}$ in dinmeter, the wire being insulated except at the end by a closely fitting glass tube. The insulated wire extended diametrically about two-thirds of the way through the anode cylinder, and the surface was viewed normally through a quartz window and a hole in the side of the cylinder. The image of the surface was focussed by a quartz 
fluorite achromat or a quar'z water achromat ${ }^{4}$ on the slit of the spectrograph.

Roughly quantitative intensity estimates are based on densitometer measurements of several spectra obtained with different exposure times assuming the reciprocity law to hold.

\section{DISCHARGE CONDITIONS}

Observations have been made with discharges in caesium, potassium, and helium with most of the measurements in caesium. An essential condition for observation of the faint continuous spectrum is the absence of strong emission lines from the discharge in the spectral region studied. In caesium wave lengths less than 3,200 $\mathrm{A}$ and between $\tilde{5}, 000$ and $4,600 \mathrm{~A}$ are particularly favorable.

For the caesium discharge, currents of the order of 1 ampere at 5 to 7 volts with pressures between 0.002 and $0.01 \mathrm{~mm}$. were used. Under these conditions most of the space between cathode and anode is at a nearly constant potential slightly positive with respect to the anode. The potential of the space is found from the current voltage characteristics of the probe. ${ }^{5}$ As one approaches the space potential from the negative side the electron current increases rapidly, while above the space potential the current remains nearly constant. On a scale of log current versus voltage the negative branch of the curve is a straight line and the slope of the line gives the average energy of the electrons in the space. The intersection of this line and the nearly horizontal positive branch gives the space potential. At positive potentials the current flow is governed by the space charge equation for parallel plate electrodes.

$$
I=2.34 \times 10^{-6} A V^{372} / x^{2}
$$

where $I$ is the current in amperes, $V$ is the potential in volts relative to the space, $A$ is the collecting area in $\mathrm{cm}^{2}$, and $x$ is the sheath thickness or distance normal to the surface to which the applied field is limited. For our conditions $I / A$ was over an ampere sc that $x$ was between $10^{-2}$ and $10^{-3} \mathrm{~cm}$. At some positive potential (5 to 20 volts, depending on the pressure) the sheath breaks down and a brilliant ball of discharge appears on the probe. At any lower potential conditions are favorable for quantitiative measurements. The average electron energy at the sheath surface is given by the semilogarithmic plot (it was always about 0.4 volts in caesium). The potential drop across the sheath is the applied potential minus the space potential. The sum gives the electron energy at the probe surface and to this should be added the work function of the metal surface if it is assumed that radiation takes place within the metal. Unfortunately the work function of a metal surface in a caesium discharge is quite uncertain and may range from 1 to 4 or 5 volts, depending on the temperature and the surface condition. The probes are heated considerably in operation, to visible red heat in extreme cases.

\section{LOCATION OF RADIATION}

The probe surface at a positive potential appears bright, but much of this light comes from the thin sheath for the radiation from the dis- 
charge is enhanced where the electrons are accelerated. The image of a fine wire probe was accurately focussed on the slit of a spectrograph. The width of the continuous spectrum from the wire at a positive potential was equal to the width of the shadow of the wire when the wire was on open circuit and definitely less than the sheath width as measured by the enhanced portion of the spectrum lines. The sheath thickness of the order of $10^{-2} \mathrm{~cm}$ was too small to measure accurately, however.

\section{OBSERVATIONS IN VARIOUS GASES}

The continuous spectrum from probes in potassium and helium discharges is qualitatively similar to that observed in caesium both in intensity and in spectral intensity distribution. Negative results were obtained in a mercury discharge where the scattered light was always sufficient to mask a continuous spectrum of the usual intensity. The observations in helium are difficult but with very pure gas there is little scattered light beyond 2,800 A. Nearly all quantitative measurements were with probes in a caesium discharge.

\section{VAPOR PRESSURE}

There is no appreciable variation of the intensity of the probe radiation in a caesium discharge with pressure in the range 0.002 to $0.01 \mathrm{~mm}$. when conditions are adjusted to keep the probe current constant.

\section{PROBE CURRENT}

The intensity is nearly proportional to the current. Spectra of equal exposure time of radiation reduced by a grid of 30 per cent transmission and of full radiation with 30 per cent of the initial current show nearly equal densities.

\section{SPECTRAL INTENSITY DISTRIBUTION}

The ultra-violet radiation from a copper probe at +7 volts with a current of $1.4 \mathrm{amp} . / \mathrm{cm}^{2}$ was compared directly with the spectrum of a calibrated tungsten strip lamp in quartz using a quartz spectrograph. Later a similar comparison was made in the visible at 6 volts and $3.1 \mathrm{amp} / \mathrm{cm}^{2}$ using a 3-prism Zeiss glass spectrograph. Figure 2 gives the results. The intensity $J(\lambda)$ remains of the same magnitude throughout the range covered and other results indicate that there is no marked decrease to $2,300 \Lambda$. The figure includes a plot of the radiation flux in quanta per unit frequency range per $\mathrm{cm} .{ }^{2}$ Ordinates give absolute values for the ultra-violet measurements with the others reduced to the same scale by an arbitrary factor. A questionable correction for scattered radiation has been applied for wave lengths longer than 5,500 A, and beyond 6,000 the curve is extremely uncertain.

\section{INTENSITY AND VOLTAGE}

The intensity changes rapidly with voltage, but between 5 and 20 volts there is very little change in the intensity distribution. Below 5 volts the intensity drops off more rapidly in the ultra-violet. Figure 3 shows the relative intensity at 2 and 3 volts as compared 


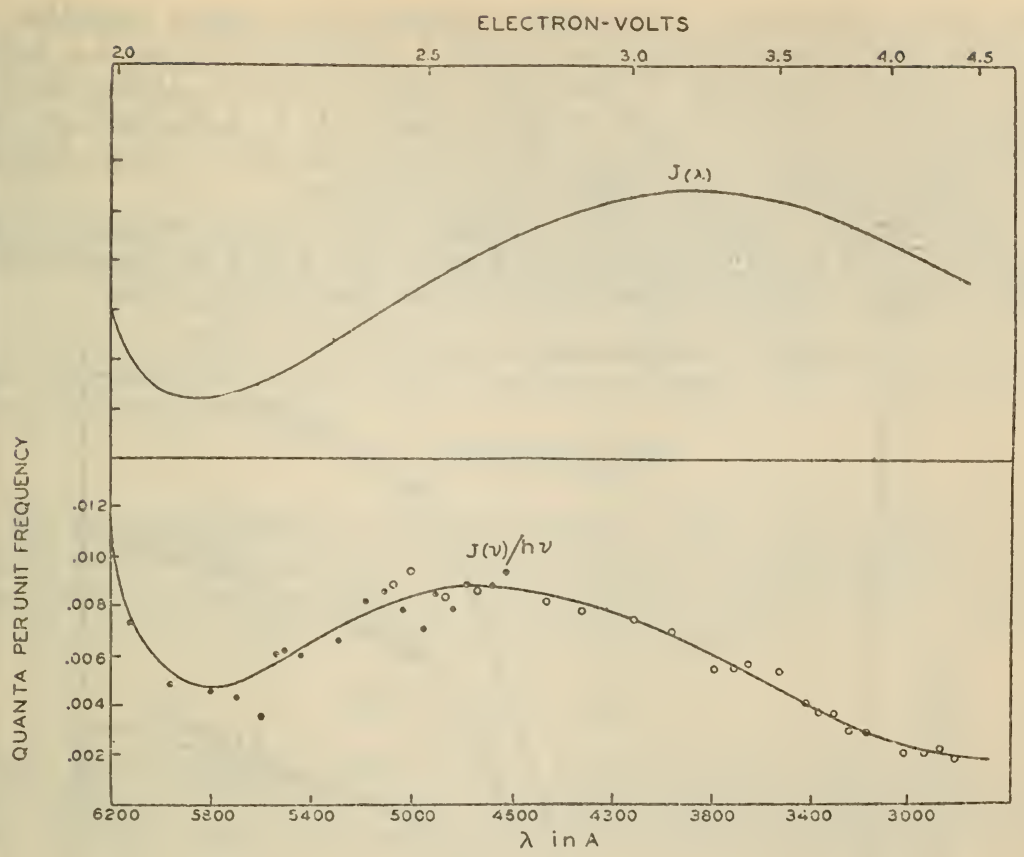

FIGURE 2.-Iniensity distribution of radiation from copper

Circles and ordinates apply to measurements with 7 volts and 1.4 amperes/ $/ \mathrm{cm}^{2}$ while dots give another sot of readings multiplied by an arbitrary factor

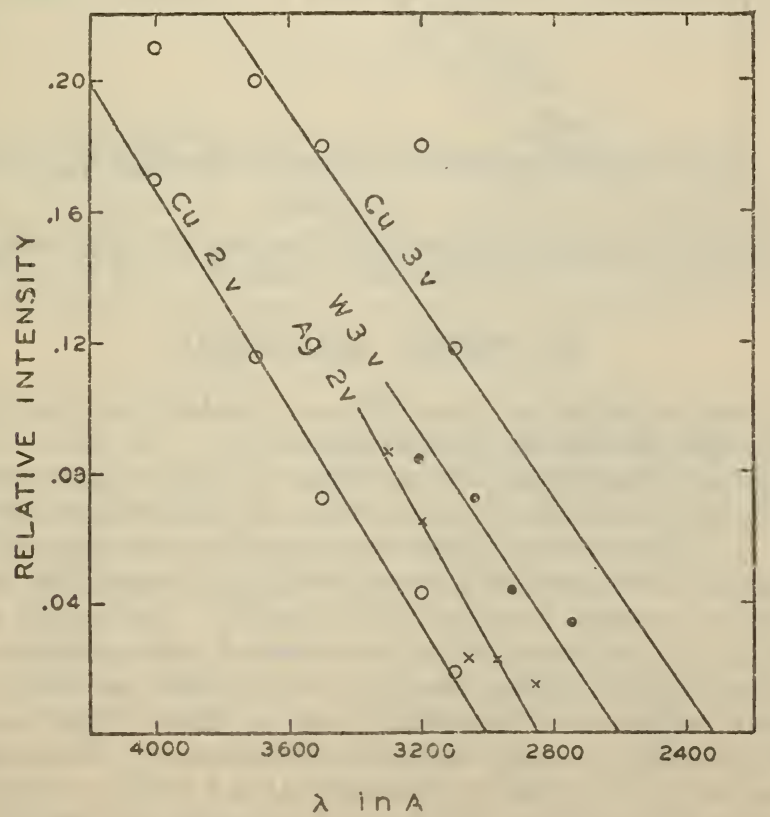

FIGURE 3.-Relative intensity at low voliages as compared with intensity at 5 volts 
with that at 5 volts. The relative intensity for copper radiation at 2 volts drops toward zero near 3,000 A corresponding to 4.1 volts. The corrected potential $\mathrm{V}$ was $1.75+.4=2.15$ volts. Using the relation $h \nu_{0}=e(V+W)$, the work function $W$ is 1.95 volts. The threshold for copper radiation with 3 -volt electrons is less definite but approximately 1 volt higher, as it should be.

The intensity variation with voltage at any wave length is characterized by a rapid increase up to 7 volts and a slow increase beyond this. Figure 4 shows the isochromats for three wave lenths.

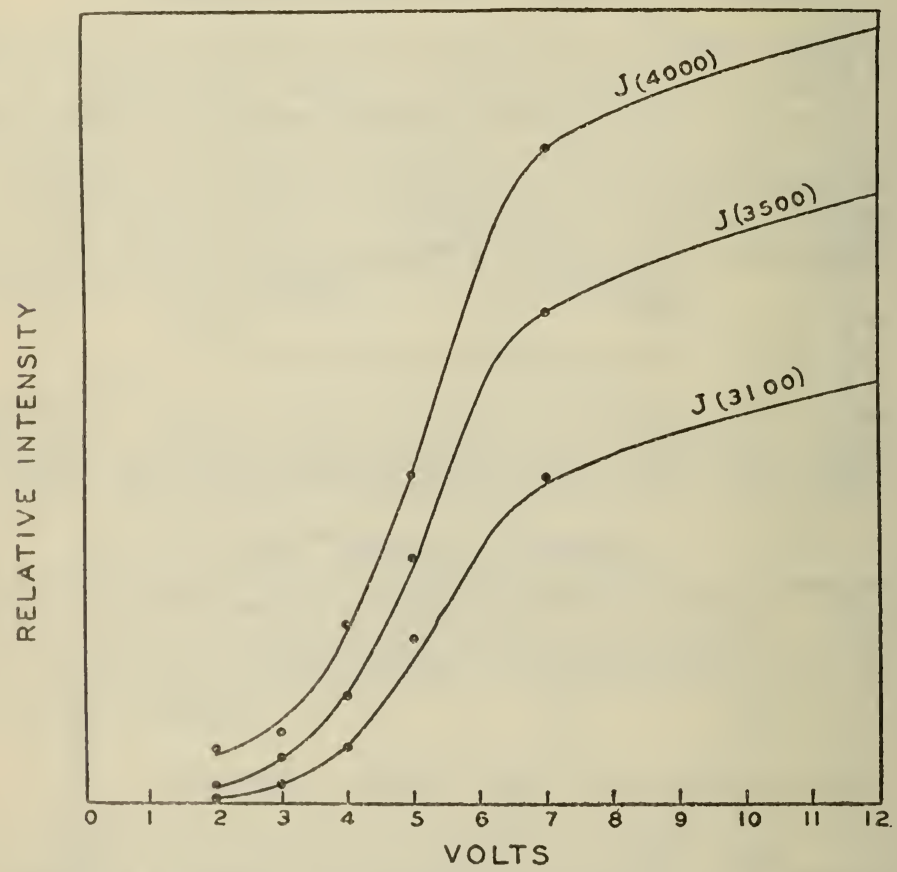

FIGURE 4.-Relative monochromatic intensity of copper radiation as a function of voltage

\section{PROBE MATERIAL}

Comparison of radiation from different metals was made by having two probes side by side in the discharge.

Silver gives radiation five or six times as bright as copper between 2,680 and $3,300 \mathrm{~A}$ and nearly twenty-five times as bright between 3,500 and $4,000 \Lambda$. The relative intensity at 2 volts as compared to that at 5 volts is much the same as for copper (fig. 3) and the threshold at $2,860 \Lambda$ gives a work function of 2.1 volts. The probe surface appeared bright in the vacuum and was coated with a white oxide when air was admitted indicating that the surface was an alloy.

Tungsten radiation is probably two or three times as bright as copper with much the same intensity distribution. Figure 3 includes measurements of the relative intensity at 3.3 volts as compared with 5 volts. The threshold at $2,600 \mathrm{~A}$ corresponds to a work function of 1.45 volts. 
Platinum was compared with a tungsten surface which was at first slightly oxidized. The tungsten radiation at 7 volts was at first weak and gradually increased under heavy electron bombardment. Finally the platinum radiation at 7 volts was weaker than the tungsten, ranging from 0.5 times tungsten at $3,200 \mathrm{~A}$ to 0.3 times tungsten at $2,500 \mathrm{~A}$. At 3 volts applied potential the situation was reversed with the platinum radiation much stronger, particularly in the uitra-violet. The platinum intensity at 3 volts as compared with 7 volts remained about 0.2 between 3,200 and $2,500 \mathrm{~A}$. The tungsten ratio dropped from 0.04 at 3,200 A toward zero near 2,600 A. The result indicates that the platinum has a work function at least a volt or two greater than the tungsten.

\section{DISCUSSION}

We have suggested in the introduction that the radiation from probe surfaces is analogous to the continuous X-ray spectrum. In the $\mathrm{X}$-ray range the intensity distribution of radiation from a thick target can be roughly represented by the equation

$$
J(\nu) d \nu=c_{1} Z\left(\nu_{0}-\nu\right) d \nu
$$

where $Z$ is the atomic number and $\nu_{0}$ is given by the relation $h \nu_{0}=V e$. The efficiency $E^{6}$; that is, the total radiant energy divided by the electron energy, based on experimental values of $c_{1}$ in equation (1), is

$$
E=9.5 \times 10^{-10} Z V \text { (volts) }
$$

The isochromatic energy as a function of $V$ is approximately

$$
J(\nu)=c_{2} Z\left(V-V_{0}\right)
$$

where $V_{0}$ is the threshold value of $V$.

Kramers ${ }^{7}$ has derived the above relations on the basis of the correspondence principle, assuming that a quantum is emitted when the impacting electron makes a transition between hyperbolic orbits in the field of an atomic nucleus. The theoretical constants are nearly identical with the experimental values and equation (1) is given in the form

$$
J(\nu)(\mathrm{ergs})=i \times 5 \times 10^{-50} Z\left(\nu_{0}-\nu\right)
$$

where $i$ is the number of electrons striking the surface per second.

The above results apply to radiation produced in the metal corrected for absorption. A reduction of our observations to intensities in the metal involves unknown factors. We can assume that radiation takes place at an average depth equal to the mean free path of an electron in the metal. Estimates of this, based on photo-electric properties of thin films, range from 2.7 to $25 \times 10^{-7} \mathrm{~cm}^{8}$ Ultraviolet radiation is reduced to $1 / e$ in about $20 \times 10^{-7} \mathrm{~cm}$ in all the metals used. ${ }^{9}$ Thus electrons are stopped at depths from which radiation can emerge and the measured effects probably come from

\footnotetext{
Int. Crit. Tables, 6, p. 46.

7 Kramers, Phil. Mag., 46, p. 836; 1923.

8 Gudden, Lichtelektrische Erscheinungen, p. 78. Julius Springer.

$\checkmark$ Int. Crit. Tables, 5, p. 248.
} 
electrons of speeds varying from the initial speed to zero. The radiation is modified more or less by absorption but not in order of magnitude. This is qualitatively similar to the case of hard $X$ rays from thick targets.

The intensity measurements for radiation from a copper probe with $1.4 \mathrm{amp}$. per $\mathrm{cm}^{2}\left(0.91 \times 10^{19}\right.$ electrons $\left./ \mathrm{cm}^{2}\right)$ at 7 volts $(V+W=$ 9.1 volts, $\left.\nu_{0}=22 \times 10^{14}\right)$ give a radiation flux at $3,700 \mathrm{~A}$ of $J(\nu)=$ $3 \times 10^{-14}$ ergs per $\mathrm{cm}^{2}$ per sec. per unit frequency range. For these conditions equation $(4)$ gives $J(\nu)=1.85 \times 10^{-14}$ ergs. For radiation of 2 -volt electrons at 1 volt from the threshold $(4,000 \mathrm{~A})$ both experiment and equation (4) give about one-sixth of the above values.

Assuming $J(\nu)$ at 3,700 to be a mean value over the range $\nu_{0}$ to 0 , the total energy radiated is $66 \mathrm{erg} \mathrm{s}$ per $\mathrm{cm}^{2}$ per sec. and the efficiency $5 \times 10^{-7}$. Equation (2) gives $2.5 \times 10^{-7}$.

The above agreements are better than we have any reason to expect. In particular we may question whether it is legitimate to use the atomic number $Z$ in these equations when applied to low-speed electrons.

One can make a qualitative comparison of the intensity we have observed for copper with the intensity reported by Foote, Meggers, and Chenault ${ }^{10}$ on the basis of equation (3). They used a current density of about $0.01 \mathrm{amp} . / \mathrm{cm}^{2}$ at 1,000 volts and photographed the continuous spectrum in about five hours. We used $1.35 \mathrm{amp} . / \mathrm{cm}^{2}$ at 7 volts applied potential, and the exposure time with a much wider slit was about hali an hour. The intensity per unit current was of the order of 50 imes greater at 1,000 volts. Equation (3) with $V-V_{0} 5$ volts and 1,000 volts gives a ratio of 200 .

A rough comparison with the measured photo-electric effect of the radiation produced by low voltage electron bombardment is possible. The photo-electric current from platinum produced by 200 volt copper radiation is $1.5 \times 10^{-6}$ times the bombarding current. ${ }^{11}$ The effeet at 5 volts applied potential $(V+W=10)$ is perhaps $3 \times 10^{-3}$ times this. ${ }^{12}$ The photo-electric efficiency of quanta between 5 and 10 electron volts may be taken as $2 \times 10^{-3}$ so that $2.2 \times 10^{-6}$ quanta per electron are emitted in a range of $1.2 \times 10^{-15}$ frequency units or $2 \times 10^{-21}$ quanta per electron per unit frequency range. The average value found in the near ultra-violet was $6 \times 10^{-22}$.

The observed radiation process is similar to, but not identical with, the converse of the photo-electric effect. Fowler ${ }^{13}$ gives a relation derived by the principle of detailed balance between the probability $\phi$ of photo-electric ejection of an electron with energy $\eta$ by a quantum $h \nu$ and the probability $\phi^{\prime}$ of emission of $h \nu$ by an electron of energy $\eta$ assuming both processes governed by the ideal relation

$$
h \nu=W e+\eta
$$

where $W$ is the work function of the metal.

$$
\phi=\frac{m c^{2} \eta}{h^{2} \nu^{2}} \phi^{\prime}
$$

10 Foote, Meggers, and Chonault, J. Opt. Soc. Am., 9, p. 541; 1924.
11 Bandopadhyaya, Proc. Roy. Soc., A120, p. 46; 1928.

12 Compton and Thomas, Phys. Rev., 28, p. 601; 1926.

13 Fowler, Statistical Mechanics, p. 494 . Cambridgo Press. 
It may be permissible to compare the number of electrons of all energies less than $\eta$ ejected by a frequency $\nu$ with the number of quanta of all frequencies less than $\nu$ excited by electrons of energy $\eta$. From measurements of 2 volt copper radiation we estimate the total radiation to be about $1.3 \times 10^{-7}$ quanta per electron. An extrapolation of photo-electric data ${ }^{14}$ for copper gives the emission by quanta of energy $(W+2)$ e as $10^{-3}$ electrons per quantum. Equacion (6) with values of $\nu$ and $\eta$ appropriate for the radiation measurements gives $\phi=6 \times 10^{4} \phi^{\prime}=8 \times 10^{-3}$ electrons per quantum as compared with $1 \times 10^{-3}$ observed. The agreement in order of magnitude is interesting in spite of the fact that the equation applies to an ideally simplified case. Equation (6) expresses the balance between photoelectric emission and radiation from electron bombardment from the metal as a whole and not from atoms in the metal in contrast with the $\mathrm{X}$-ray equations.

Washington, February 14, 1931.

1s Gudden, Lichtelekstrische Erscheinungen, p. 23. Julius Springer.

$43321^{\circ}-31-11$ 